\title{
Secondary grating formation by readout at Bragg-null incidence
}

\author{
Ali Adibi, Jose Mumbru, Kelvin Wagner, and Demetri Psaltis
}

\begin{abstract}
We show that when a dynamic hologram is read out by illumination at the Bragg nulls of a previously recorded grating the diffracted beam inside the medium can result in the recording of two secondary gratings that alter the final selectivity curve. This is confirmed experimentally. This effect can cause cross talk in hologram multiplexing that is stronger than interpage cross talk when a small number of holograms with high diffraction efficiencies are multiplexed. (C) 1999 Optical Society of America

OCIS codes: $090.0090,210.2860,090.2910$.
\end{abstract}

\section{Introduction}

The angular selectivity curve of a grating recorded in a photorefractive medium shows angles at which the output diffraction efficiency is very small. These angles, which are called Bragg nulls, are important when holograms are angle multiplexed. Adjacent holograms are usually recorded at the nulls of their neighbors to minimize cross talk. In this paper we investigate the modification that takes place in a grating when it is illuminated at its Bragg nulls. The effect on cross talk when multiple holograms are recorded is also discussed.

\section{Theory}

For a sinusoidal index grating (shown in Fig. 1) the permittivity inside the medium can be represented as

$$
\Delta \epsilon=\Delta_{0} \exp \left(j K_{g} x\right) \operatorname{rect}\left(\frac{z-L / 2}{L}\right)
$$

where $K_{g}$ is the magnitude of the grating vector and $\Delta_{0}$ is a constant representing the strength of the grating. When the grating is read out by a plane wave $E_{i}$ $=A_{i} \exp \left(j \mathbf{k}_{i} \cdot \mathbf{r}\right)$, the diffracted field inside the me-

The authors are with the Department of Electrical Engineering, Pasadena, California 91125. D. Psaltis' e-mail address is psaltis@sunoptics.caltech.edu.

Received 19 January 1999; revised manuscript received 26 March 1999.

0003-6935/99/204291-05\$15.00/0

(C) 1999 Optical Society of America dium with infinite transverse dimensions is given under the Born approximation ${ }^{1}$ by

$$
\begin{aligned}
E_{d}(x, z)= & \frac{\alpha A_{i}}{j} \exp \left(j \mathbf{k}_{d} \cdot \mathbf{r}\right) \\
& \times\left[\exp \left(-j \Delta k_{z} z / 2\right) \frac{\sin \left(\Delta k_{z} z / 2\right)}{\Delta k_{z} L / 2}\right],
\end{aligned}
$$

where $\alpha$ is the amplitude diffraction efficiency of the original grating and $\mathbf{k}_{d}$ represents the diffracted field wave vector inside the medium as shown in Fig. 2. $\Delta k_{z}=k_{d z}-k_{i z}$, with $k_{i z}$ and $k_{d z}$ being the $z$ components of $\mathbf{k}_{i}$ and $\mathbf{k}_{d}$, respectively. Bragg nulls occur at the input angles where the output field $E_{d}(x, L)$ is zero. This happens at $\Delta k_{z p}=(2 p \pi / L)$, with integer $p$ representing the $p$ th null. Reading out the grating at its $p$ th null with a plane wave $E_{i}=A_{i} \exp \left(j \mathbf{k}_{i} \cdot \mathbf{r}\right)$ results in the following diffracted field inside the medium:

$$
\begin{aligned}
E_{d p} & =\frac{\alpha A_{i}}{j} \exp \left(j \mathbf{k}_{d p} \cdot \mathbf{r}\right)\left\{\exp \left(-j \frac{p \pi}{L} z\right) \frac{\sin [(p \pi / L) Z]}{p \pi}\right\} \\
& =\frac{\alpha A_{i}}{2 p \pi} \exp \left(j \mathbf{k}_{d p} \cdot \mathbf{r}\right)\left[\exp \left(-j \frac{2 p \pi}{L} z\right)-1\right]
\end{aligned}
$$

where $\mathbf{k}_{d p}$ is the wave vector of the diffracted field for the $p$ th null readout.

The variation of the diffracted field amplitude inside the medium for illumination at the first two nulls along with that of the Bragg-matched readout is depicted in Fig. 3. Although the output field is zero for the null incidence angle, the field inside the medium is not. The simultaneous presence of this internal diffracted field and the incident (reading) beam re- 


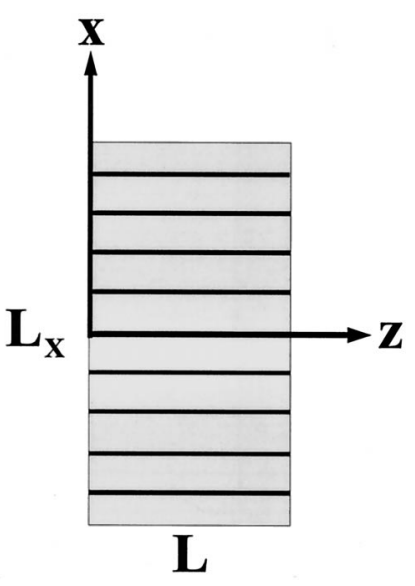

Fig. 1. Geometry of a typical grating recorded in a photorefractive crystal in a symmetric transmission geometry. The $c$ axis of the crystal is parallel to the $x$ axis.

sults in an inhomogeneous light intensity pattern inside the medium given by

$$
\begin{aligned}
I= & \left|E_{d p}+E_{i}\right|^{2} \approx I_{i}\left\{1+\frac{\alpha}{p \pi}\left[\cos \left(K_{g} x\right)\right.\right. \\
& \left.\left.-\cos \left(K_{g} x+\frac{2 p \pi}{L} z\right)\right]\right\},
\end{aligned}
$$

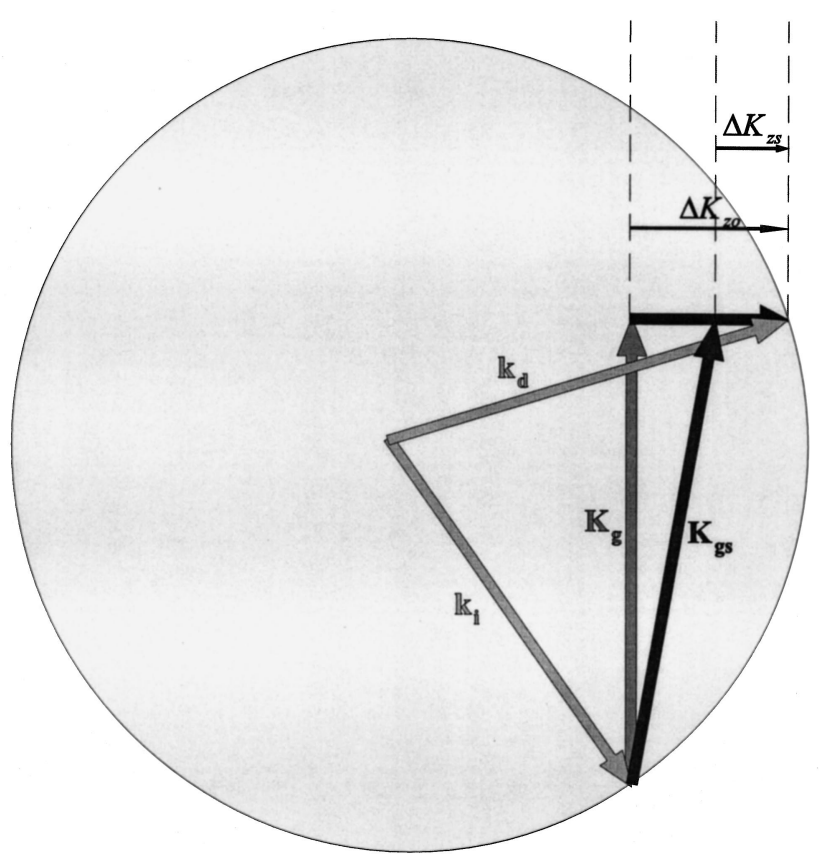

Fig. 2. Readout of the original and the secondary gratings. $\mathbf{k}_{i}$ and $\mathbf{k}_{d}$ represent the wave vectors of the incident and the diffracted beams, respectively. $\mathbf{K}_{g}$ and $\mathbf{K}_{g s}$ are the original and the secondary grating vectors, respectively. The Bragg mismatch for the original and the secondary gratings are shown by $\Delta k_{z o}$ and $\Delta k_{z s}$, respectively. The mismatch is nonzero only in the $z$ direction, since we assumed infinite dimensions in the other directions for the grating. It is clear from this figure that the Braggmatched angles for these two gratings are different.

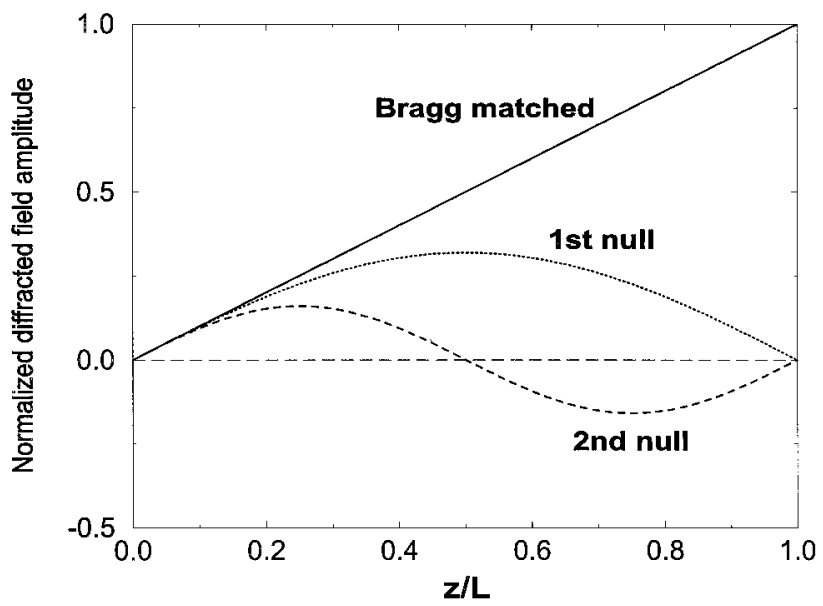

Fig. 3. Variation of the normalized diffracted field amplitude with the position inside the grating medium for the Bragg-matched and the first two null exposures. The curves are normalized to the Bragg-matched diffracted beam amplitude and not to the input beam amplitude.

with $I_{i}$ being the intensity of the reading beam. This results in the recording of two secondary gratings that alter the angular selectivity curve. The strength of these secondary gratings depends on the recording time and the intensity of the incident beam. If we assume the same average intensities and recording times for the original and the secondary gratings, the overall diffracted field at the output plane $(z=L)$ can be calculated with the Born approximation as

$$
\begin{aligned}
E_{d} \sim & {\left[\left(1+\frac{\beta}{p}\right) \operatorname{sinc}\left(\frac{\Delta k_{z} L}{2 \pi}\right)+(-1)^{p} \frac{\beta}{p}\right.} \\
& \left.\times \operatorname{sinc}\left(\frac{\Delta k_{z} L}{2 \pi}-p\right)\right] \exp \left(j \mathbf{k}_{d} \cdot \mathbf{r}\right),
\end{aligned}
$$

where $\beta$ represents the relative strength of the secondary gratings and is given by $\beta=(\alpha / \pi m)$, with $m$ being the modulation depth of the intensity pattern that records the original grating. Here we assumed that charge transport in the medium is dominated by bulk photovoltaic effect. ${ }^{2}$ In such media the recorded space-charge field and the intensity pattern are either in phase or $180^{\circ}$ out of phase, resulting in a real-valued $\beta$. This is the case in $\mathrm{LiNbO}_{3}$, for example.

The readout at the $p$ th null results in the recording of two secondary gratings, one with the same grating vector as the original grating and the other being Bragg matched to the $p$ th null incident beam. This is depicted in Fig. 2. The diffraction efficiency as a function of readout angle is shown in Fig. 4 for gratings recorded and subsequently illuminated at their 1st, 2nd, and -2nd nulls. Notice that the selectivity curve for the 1st null has a pronounced asymmetry, with the first sidelobe being much larger in one direction. This is because the two sinc functions in relation (5) add in phase in one direction and out of 


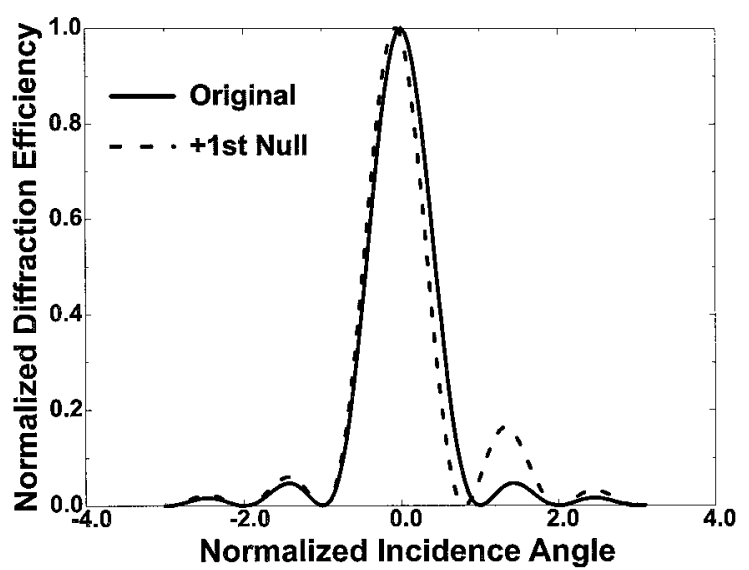

(a)

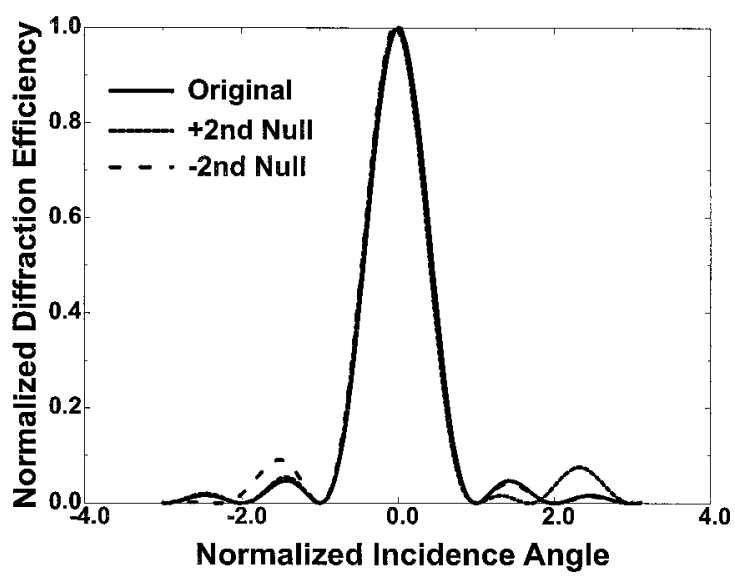

(b)

Fig. 4. Normalized theoretical selectivity curves for the grating: (a) after exposures at the first right null, (b) after exposures at the second nulls for both right- and left-hand sides. The selectivity curve of the original grating (before exposure) is also shown for comparison. All curves are normalized to have the same maximum value as that of the original grating. The reference for $0^{\circ}$ is the Bragg-matched angle.

phase in the other. Note that the peaks of the sidelobes are shifted from their original positions.

\section{Experiments}

To verify these theoretical results, we performed recording experiments with a 4-mm-thick $\mathrm{LiNbO}_{3}$ crystal doped with 0.015 wt. $\% \quad \mathrm{Fe}_{2} \mathrm{O}_{3}$, using the symmetric transmission geometry with two beams of wavelength $488 \mathrm{~nm}$ from an argon-ion laser. Both beams had the same diameter $(0.4 \mathrm{~cm})$ and intensity $\left(9 \mathrm{~mW} / \mathrm{cm}^{2}\right)$. The polarization of the beams was ordinary, and the angle between them outside the crystal was $30^{\circ}$. First, we recorded a grating with $10 \%$ diffraction efficiency and measured its selectivity curve. Next, one of the recording beams was blocked and the angle of the other beam set to one of the Bragg nulls, and illumination at the null was performed for $50 \mathrm{~min}$, while the buildup of diffraction efficiency with time was monitored. Finally, the selectivity curve was measured again and compared

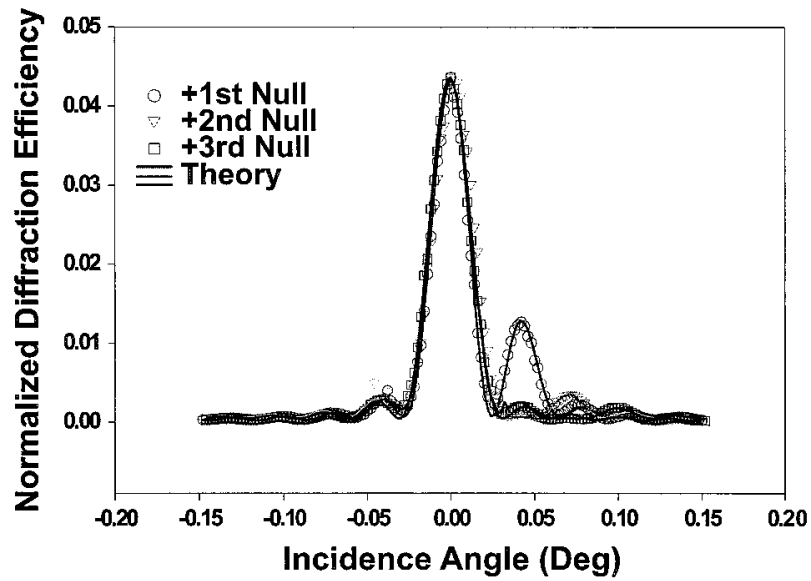

(a)

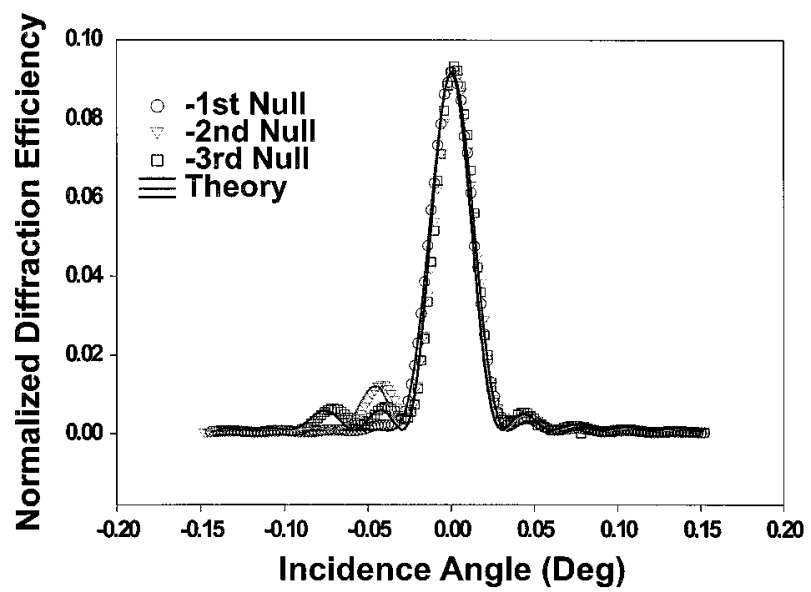

(b)

Fig. 5. Normalized theoretical and experimental selectivity curves for the grating after 50 min of exposure at one of its Bragg nulls for (a) the first three right nulls and (b) the first three left nulls. The original grating was recorded to $10 \%$ diffraction efficiency. Solid curves represent theoretical fits. All curves are normalized to have the same maximum value and position as that of the original grating. The reference for $0^{\circ}$ is the Bragg-matched angle.

with that before the null incidence. These steps were repeated with a freshly erased crystal for different nulls. The experimental angular selectivity curves are depicted in Fig. 5. The selectivity curves were normalized to result in the same Braggmatched peak and location. Solid curves represent theoretical fits. We had to adjust the relative position of the two sinc functions in relation (5) to get the best fit to the experimental results. The maximum deviation from an integer $p$ for the best fit was $20 \%$. The deviations for all nulls were in one direction, thus suggesting the possibility of Bragg shift of the secondary gratings, owing to two-beam phase coupling, which produces tilted and possibly curved fringes. ${ }^{3,4}$ Figure 5 confirms the asymmetric boost that is expected from theory. The recording curves for the secondary gratings are depicted in Fig. 6 for the first 


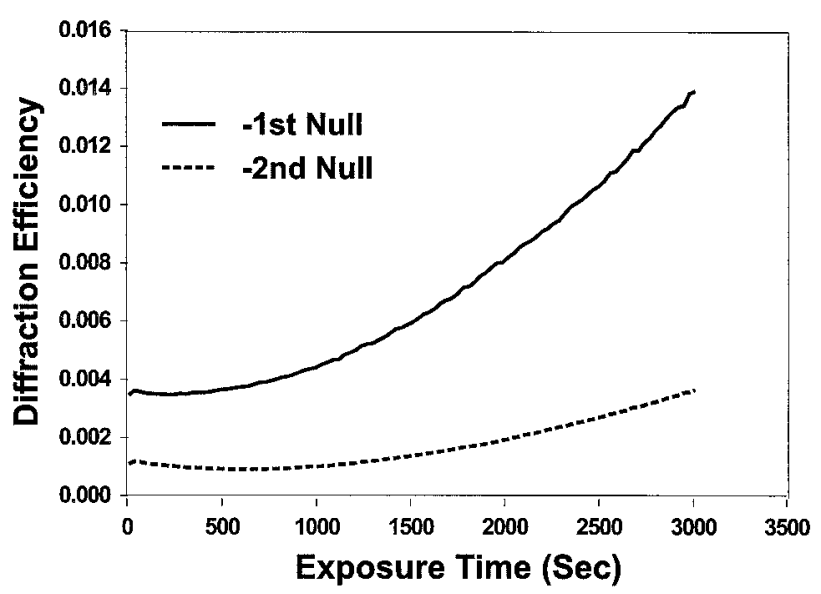

Fig. 6. Recording curves for the secondary gratings recorded by reading of the original grating at its first two left nulls.

two left nulls. It should be noted that, owing to the erasure of the original grating, which is the source of the secondary gratings, during this process, the curves are not represented by simple monoexponential formulas. Finally, Fig. 7 depicts the relative strength of the secondary gratings $(\beta / p)$, found when we fit relation (5) to the experimental results for the first three nulls. The $1 / p$ behavior suggested by the theory is clear.

\section{Application to Cross Talk in Holographic Memories}

The secondary holograms can cause cross talk in hologram multiplexing where each hologram is recorded at the Bragg nulls of the previous ones. While each hologram is being recorded, the reference beam will diffract off the previous holograms, resulting in the recording of secondary holograms that, although weak, are Bragg matched to the readout beam of the present hologram. This results in cross talk among different holograms. ${ }^{5}$ To compare this cross-talk mechanism with the well-known interpage crosstalk, ${ }^{5-7}$ we calculate the noise-to-signal ratio (NSR) for the $90^{\circ}$ geometry system shown in Fig. 8.

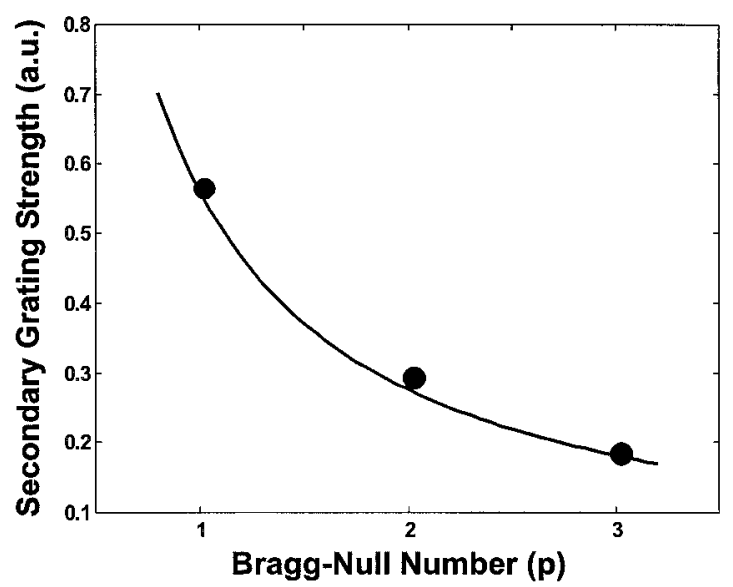

Fig. 7. Strength of the secondary gratings for the first three right nulls. Curve, $1 / n$ fit as theoretically expected.

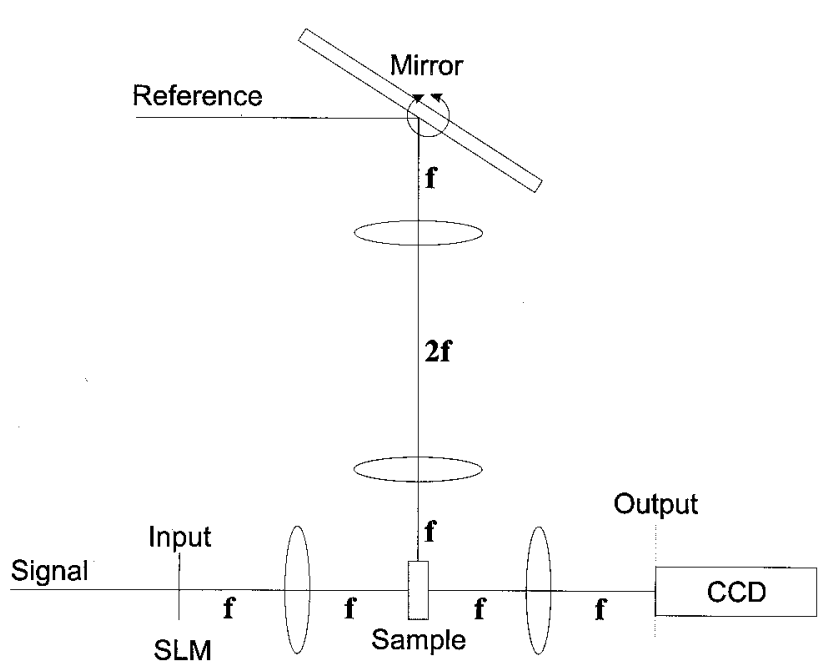

Fig. 8. Typical 4-f system for multiplexing holograms with anglemultiplexed $90^{\circ}$ geometry.

The focal lengths of all lenses $(f)$ are taken to be 30 $\mathrm{cm}$, and the size of the input image is assumed to be $1 \mathrm{~cm} \times 1 \mathrm{~cm}$. To record $M$ holograms with equal diffraction efficiencies, a recording schedule ${ }^{8}$ can be used, resulting in holograms with equal diffraction efficiencies $(\eta)$, each with $\eta=(M / \# / M)^{2}$. We assume the crystal to be $1 \mathrm{~cm}$ thick with $M / \#=1.3$ and the modulation depth of the recording intensity profile to be $m=0.8$ for the cross-talk calculation. To calculate the worst-case cross talk, we assume that each hologram is recorded at the first null of the previous one. This calculation was performed by numerical evaluation of the secondary grating cross talk that was analytically derived. The analysis took into account all possible contributions to secondary cross talk and the effect of the exposure schedule. The details of the analysis will be presented elsewhere. Figure 9(a) shows the maximum NSR as a function of hologram position for 101 holograms (numbered from 1 to 101). Cross-talk noise is a nonconstant two-dimensional function of the coordinates in the output plane. In comparing different types of cross talk, we calculate the NSR in each case at the location in the output plane with the largest crosstalk noise. Figure 9(a) suggests that secondary cross talk depends strongly on hologram position. This is due to the recording schedule. The initial holograms are recorded to higher diffraction efficiencies and for a longer time, resulting in stronger secondary holograms (except for the first hologram, which does not suffer from secondary cross talk). It can also be said that, for a small number of holograms, secondary cross talk is more important than interpage cross talk for at least the first half of the holograms. When we increase the number of holograms, maximum interpage cross talk becomes stronger, whereas secondary cross talk becomes weaker, owing to smaller diffraction efficiencies and shorter recording times involved. For example, if 240 holograms are multiplexed with the same system, interp- 


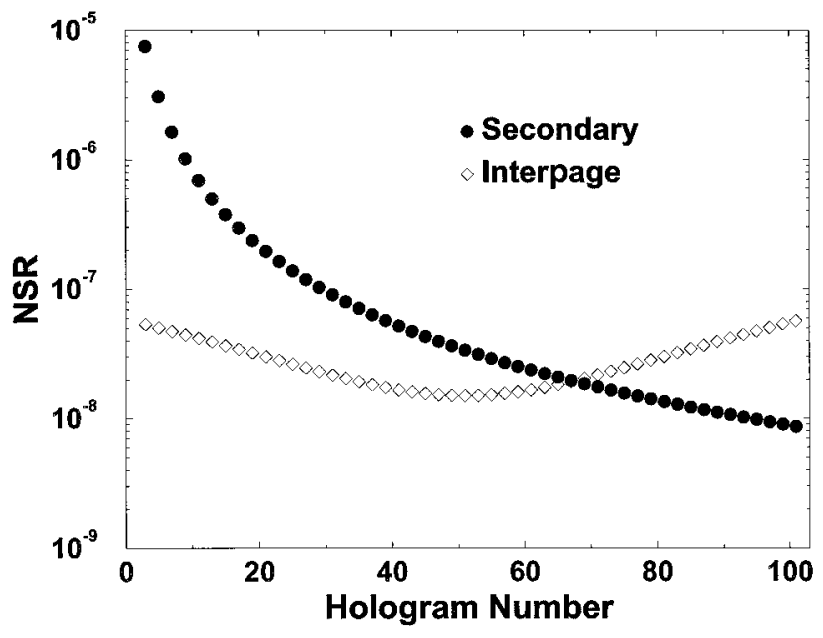

(a)

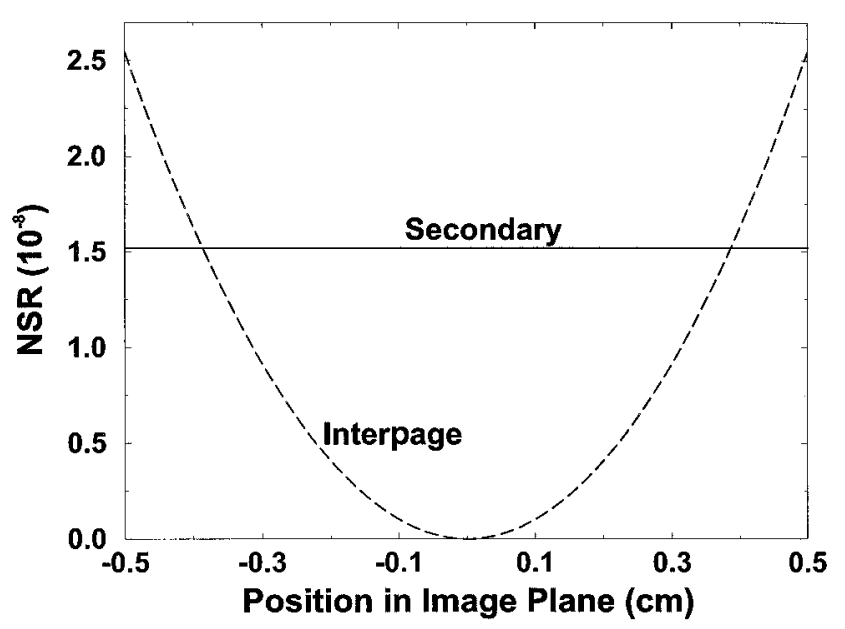

(b)

Fig. 9. Comparison of secondary and interpage cross talk. Theoretical NSR when $M=101$ holograms are recorded with the system of Fig. 8. (a) NSR for different holograms. (b) NSR versus location in the output plane for hologram number 76 .

age cross talk becomes dominant for all holograms. Figure 9(b) depicts the variation of NSR within the output plane for the 76th hologram. It is clear that secondary cross talk is almost the same throughout the image, whereas interpage cross talk shows much larger variation with position. Therefore there are positions at the output plane at which secondary cross talk becomes more important even for a large number of holograms. Finally, it should be noted that secondary cross talk can also build up during readout, especially for strong readout beams.

\section{Conclusions}

In conclusion, we have presented a theoretical analysis and experimental verification of the recording of two secondary gratings that are due to the readout of a grating at its Bragg nulls. One of these gratings is the same as the original and the other one is Bragg matched to the null incident beam. The relative strength of these secondary gratings is linearly proportional to the field diffraction efficiency of the original grating and inversely proportional to the order of the Bragg null. Finally, we have showed that, in multiplexing holograms, this effect results in cross talk among different holograms that can be stronger than interpage cross talk for a small number of holograms with high diffraction efficiencies.

This study was funded by Air Force/Rome Lab award F0060297C0049.

\section{References}

1. H.-Y. S. Li, "Photorefractive 3-D disks for optical data storage and artificial neural networks," Ph.D. dissertation (California Institute of Technology, Pasadena, Calif., 1993).

2. A. M. Glass, D. von der Linde, and T. J. Negran, "High-voltage bulk photovoltaic effect and photorefractive process in $\mathrm{LiNbO}_{3}$," Appl. Phys. Lett. 25, 233-235 (1974).

3. J. M. Heaton, P. A. Mills, E. G. S. Paige, and L. Solymar, "Diffraction efficiency and angular selectivity of volume phase holograms recorded in photorefractive materials," Opt. Acta 31, 885-901 (1984).

4. S. Tao, Z. H. Song, and D. R. Selviah, "Bragg-shift of holographic recording in photorefractive $\mathrm{Fe}: \mathrm{LiNbO}_{3}$ crystals," Opt. Commun. 108, 144-152 (1994).

5. C. Gu and J. Hong, "Noise gratings formed during the multiple exposure schedule in photorefractive media," Opt. Commun. 93, 213-218 (1992).

6. W. J. Burke and P. Sheng, "Crosstalk noise from multiple thickphase holograms," J. Appl. Phys. 48, 681-685 (1977).

7. P. Varga and G. Kiss, "Crosstalk and loss of information in holography," Kvant. Elektron. (Moscow) 10, 111-119 (1983).

8. D. Psaltis, D. Brady, and K. Wagner, "Adaptive optical networks using photorefractive crystals," Appl. Opt. 27, 1752-1759 (1988). 